\title{
Combining Local Feature Histograms of Different Granularities ${ }^{\star}$
}

\author{
Ville Viitaniemi and Jorma Laaksonen \\ Department of Information and Computer Science, Helsinki University of Technology, \\ P.O. Box 5400, FI-02015 TKK, Finland \\ \{ville.viitaniemi,jorma.laaksonen\}@tkk.fi
}

\begin{abstract}
Histograms of local features have proven to be powerful representations in image category detection. Histograms with different numbers of bins encode the visual information with different granularities. In this paper we experimentally compare techniques for combining different granularities in a way that the resulting descriptors can be used as feature vectors in conventional vector space learning algorithms. In particular, we consider two main approaches: fusing the granularities on SVM kernel level and moving away from binary or hard to soft histograms. We find soft histograms to be a more effective approach, resulting in substantial performance improvement over single-granularity histograms.
\end{abstract}

\section{Introduction}

In supervised image category detection the goal is to predict whether a novel test image belongs to a category defined by a training set of positive and negative example images. The categories can correspond, for example, to the presence or absence of a certain object, such as a dog. In order to automatically perform such a task based on the visual properties of the images, one must use a representation for the properties that can be extracted automatically from the images.

Histograms of local features have proven to be powerful image representations for image classification and object detection. Consequently their use has become commonplace in image content analysis tasks. This paradigm is also known by the name Bag of Visual Words (BoV) in analogy with the successful Bag of Words paradigm in text retrieval. In this analogue, images correspond to documents and different local feature values to words.

Use of local image feature histograms for supervised image classification and characterisation can be divided into several steps:

1. Selecting image locations of interest.

2. Describing each location with suitable visual descriptors (e.g. SIFT).

3. Characterising the distribution of the descriptors within each image with a histogram.

\footnotetext{
* Supported by the Academy of Finland in the Finnish Centre of Excellence in Adaptive Informatics Research project.
} 
4. Using the histograms as feature vectors representing the images in a supervised vector space algorithm, such as SVM.

All parts of the BoV pipeline are subject of continuous study. However, for this paper we regard the beginning of the chain (steps 1, 2 and partially also 3 ) as given. The alternative techniques we describe and evaluate in the subsequent sections place themselves and extend the step 3 of the pipeline. They can be regarded as different histogram creation and post-processing techniques that build on top of the readily-existing histogram codebooks used in our baseline implementation. Step 4 is once again regarded as given for the current studies.

The process of forming histograms loses much information about the details of the descriptor distribution. Information reduction step is, however, necessary in order to be able to perform the fourth step using conventional methods. In the histogram representation the continuous distance between two visual descriptors is reduced to a single binary decision: whether the descriptors are deemed similar (i.e. fall into the same histogram bin) or not.

Selecting the number of bins used in the histograms - i.e. the histogram size directly determines how coarsely the visual descriptors are quantised and subsequently compared. In this selection, there is a trade-off involved. A small number of bins leads to visually rather different descriptors being regarded as similar. On the other other hand, too numerous bins result in visually rather similar descriptors ending up in different histogram bins and regarded as dissimilar. The latter problem is not caused by the histogram representation itself, but the desire to use the histograms as structureless feature vectors in the step 4 above so that conventional learning algorithms can be used.

Earlier [8] we have performed category detection experiments where we have compared ways to select a codebook for a single histogram representation, with varying histogram sizes. For the experiments we used the images and category detection tasks of the publicly available VOC2007 benchmark. In this paper we extend these experiments by proposing and evaluating methods for simultaneously taking information from several levels of descriptor-space granularity into account while still retaining the possibility to use the produced image representations as feature vectors in conventional vector space learning methods. In the first of the considered methods, histograms of different granularities are concatenated with weights, corresponding to a multi-granularity kernel function in the SVM. This approach is closely related to the pyramid matching kernel method of [4. We also propose two ways of modifying the histograms so that the descriptor-space similarity of the histogram bins and descriptors of the interest points are better taken into account: the post smoothing and soft histogram techniques.

The rest of this paper is organised as follows. Our baseline BoV implementation and its proposed improvements are described in Sections 2 through 5 , Section [6] details the experiments that compare the algorithmic variants. In Section 7 we summarise the experiments and draw our conclusions. 


\section{Baseline System}

In this section we describe our baseline implementation of the Bag of Visual Words pipeline of Sect. 1. In the first stage, a number of interest points are identified in each image. For these experiments, the interest points are detected with a combined Harris-Laplace detector [6] that outputs around 1200 interest points on average per image for the images used in this study. In step 2 the image area around each interest point is individually described with a 128-dimensional SIFT descriptor [5], a widely-used and rather well-performing descriptor that is based on local edge statistics.

In step 3 each image is described by forming a histogram of the SIFT descriptors. We determine the histogram bins by clustering a sample of the interest point SIFT descriptors (20 per image) with the Linde-Buzo-Gray (LBG) algorithm. In our earlier experiments 8 we have found such codebooks to perform reasonably well while the computational cost associated with the clustering still remains manageable. The LBG algorithm produces codebooks with sizes in powers of two. In our baseline system we use histograms with sizes ranging from 128 to 8192 . In some subsequently reported experiments we also employ codebook sizes 16384 and 32768 .

In the final fourth step the histogram descriptors of both training and test images are fed into supervised probabilistic classifiers, separately for each of the 20 object classes. As classifiers we use weighted C-SVC variants of the SVM algorithm, implemented in the version 2.84 of the software package LIBSVM [2]. As the kernel function $g$ we employ the exponential $\chi^{2}$-kernel

$$
g_{\chi^{2}}\left(\mathbf{x}, \mathbf{x}^{\prime}\right)=\exp \left(-\gamma \sum_{i=1}^{d} \frac{\left(x_{i}-x_{i}^{\prime}\right)^{2}}{x_{i}+x_{i}^{\prime}}\right) .
$$

The free parameters of the C-SVC cost function and the kernel function are chosen on basis of a search procedure that aims at maximising the six-fold cross validated area under the receiver operating characteristic curve (AUC) measure in the training set. To limit the computational cost of the classifiers, we perform random sampling of the training set. Some more details of the SVM classification stage can be found in 7 .

In the following we investigate techniques for fusing together information from several histograms. To provide comparison reference for these techniques, we consider the performance of post-classifier fusion of the detection results based on the histograms in question. For classifier fusion we employ Bayesian Logistic Regression (BBR) [1] that we have found usually to perform at least as well as other methods we have evaluated (SVM, sum and product fusion mechanism) for small sets of similar features.

\section{Speed-Up Technique}

For the largest codebooks, the creation of histograms becomes impractically time-consuming if implemented in a straightforward fashion. Therefore, a speedup structure is employed to facilitate fast approximate nearest neighbour search. 
The structure is formed by creating a succession of codebooks diminishing in size with the k-means clustering algorithm. The structure is employed in the nearestneighbour search of vector $\mathbf{v}$ by first determining the closest match of $\mathbf{v}$ in the smallest of the codebooks, then in the next larger codebook. This way a match is found in successively larger codebooks, and eventually among the original codebook vectors. The time cost of this search algorithm is proportional to the logarithm of the codebook size. In our evaluations, the approximative algorithm comes rather close to the full search in terms of both MSE quantisation error and category detection MAP. Despite some degradation of performance, the speed-up structure is necessary as it facilitates the practical use of larger codebooks than would otherwise be feasible. The technique of soft histogram forming (Section 5) is able to make use of such large codebooks.

\section{Multi-granularity Kernel}

In this section we describe the first one of the considered techniques for combining descriptor similarity on various level of granularity. In this technique we extend the kernel of the SVM to take into account not only a single SIFT histogram $H$, but a whole set of histograms $\left\{H_{i}\right\}$. To form the kernel, we evaluate the multigranularity distance $d_{m}$ between two images as a weighted sum of distances $d_{i}$ in different granularities $i$, i.e. evaluated by a means of the distance

$$
d_{\mathrm{m}}=\sum_{i} w_{i} d_{i}, w_{i}=N_{i}^{1 / K}
$$

The distance $d_{i}$ is evaluated as the $\chi^{2}$ distance between two histograms of granularity $i$. In the formula for weight $w_{i}, N_{i}$ is the number of bins in histogram $i$ and $K$ is a free parameter of the method that can be thought to correspond to the dimensionality of the space the histograms quantise. Value $K=\infty$ corresponds to unweighted concatenation of the histograms. The distance values $d_{\mathrm{m}}$ are used to form a kernel for SVM through exponential function, just as in the baseline technique:

$$
g_{\mathrm{m}}=\exp \left(-\gamma d_{\mathrm{m}}\right) .
$$

The described technique is related to the pyramid match kernel introduced in 4. Also there the image similarity is a weighted sum of similarities of histograms of different granularities. However, the authors of [4] use histogram intersection as the similarity measure. They use similarities directly as kernel values, leading to also the kernel being a linear combination of similarity values. In our method this is not the case. Another difference is that in [4] the descriptor space is partitioned to histogram bins with a fixed grid whereas we employ data-adaptive clustering. Furthermore, the bins in our histograms are not hierarchically related, i.e. bins in larger histograms are not mere subdivisions of the bins in smaller histograms.

The functional form of our weighting scheme is borrowed from [4. Despite the seemingly similar form of the weighting function, their weighting scheme results 
in different relative weights being assigned to distances in different resolutions. This is because their histogram intersection measure is invariant to the number of histogram bins whereas our distance measure is not.

\section{Histogram Smoothing Methods}

In this section we describe and evaluate methods that try to leverage from the knowledge that we possess of the descriptor-space similarity of the histogram bins. In the baseline method for creating histograms, two descriptors falling into different histogram bins are considered equally different, regardless of whether the codebook vectors of the histogram bins are neighbours or far from each other in the descriptor space.

\subsection{Post-smoothing}

Our first remedy is a post-processing method of the binary histograms that is subsequently denoted post-smoothing. In this method a fraction $\lambda$ of the hit count $c_{i}$ of histogram bin $i$ is spread to its $n_{\mathrm{nbr}}$ closest neighbours. Among the neighbours, the hit count is distributed in proportion to inverse squared distance from the originating histogram bin. This histogram smoothing scheme has the convenient property that it can be applied to readily created histograms without the need to redo the hit counting which is relatively time-consuming. Alternatively, this smoothing scheme could be implemented as a modification to the SVM kernel function.

\subsection{Soft Histograms}

The latter of the described methods (denoted the soft histogram method from here on) specifically redefines the way the histograms are created. Hard assignments of descriptors to histogram bins are replaced with soft ones. Thus each descriptor increments not only the hit count of the bin whose codebook vector is closest to the descriptor, but the counts of all the $n_{\text {nbr }}$ closest bins. The increments are no longer binary, but are determined as a function to the closeness of the codebook vectors of the histogram bins to the descriptor.

We evaluated several proportionality functions for distributing bin increments $\Delta_{i}$ among the $k$ histogram bins nearest to the descriptor $\mathbf{v}$ :

1. inverse Euclidean distance : $\Delta_{i} \propto\left\|\mathbf{v}_{i}-\mathbf{v}\right\|^{-1}$

2. squared inverse Euclidean distance : $\Delta_{i} \propto\left\|\mathbf{v}_{i}-\mathbf{v}\right\|^{-2}$

3. (negative) exponential of Euclidean distance : $\Delta_{i} \propto \exp \left(-\alpha \exp \frac{\left\|\mathbf{v}_{i}-\mathbf{v}\right\|}{d_{0}}\right)$

4. Gaussian : $\Delta_{i} \propto \exp \left(-\alpha_{\mathrm{g}} \frac{\left\|\mathbf{v}_{i}-\mathbf{v}\right\|^{2}}{d_{0}^{2}}\right)$

Here the normalisation term $d_{0}$ is the average distance between two neighbouring codebook vectors. 


\section{Experiments}

\subsection{Category Detection Task and Experimental Procedures}

In this paper we consider the supervised image category detection problem. Specifically, we measure the performance of several algorithmic variants for the task using images and categories defined in the PASCAL NoE Visual Object Classes (VOC) Challenge 2007 collection [3]. In the collection there are altogether 9963 photographic images of natural scenes. In the experiments we use the half of them (5011 images) denoted "trainval" by the challenge organisers.

Each of the images contains at least one occurrence of the defined 20 object classes, including e.g. several types of vehicles (bus,car,bicycle etc.), animals and furniture. The presences of these objects in the images were manually annotated by the organisers. In many images there are objects of several classes present. In the experiments (and in the "classification task" of VOC Challenge) each object class is taken to define an image category.

In the experiments the 5011 images are partitioned approximately equally into training and test sets. Every experiment was performed separately for each of the 20 object classes. The category detection accuracy is measured in terms of non-interpolated average precision (AP). The $\mathrm{AP}$ values were averaged over the 20 object classes and six different train/test partitionings. The resulting average MAP values tabulated in the result tables had $95 \%$ confidence intervals of the order 0.01 in all the experiments. This means that, for some pairs of techniques with nearly the same MAP values, the order of superiority can not be stated very confidently on basis of a single experiment. However, in the experiments the discussed techniques are usually evaluated with several different histogram codebook sizes and other algorithmic variations. Such experiment series usually lead to rather definitive conclusions. Moreover, because of systematic differences between the six trials, the confidence intervals arguably underestimate the reliability of the results for the purpose of comparing various techniques. The variability being similar for all the results, we do not clutter the tables of results with confidence intervals.

The row $\chi^{2}$ of Table 1 summarises the category detection performance of the baseline system for different codebook sizes. A fact worth noting is that for the baseline histograms, the performance seemingly saturates at codebook size around 4096 and starts to degrade for larger codebooks.

Our multi-granularity kernel employs the $\chi^{2}$ distance measure whereas histogram intersection is used in [4]. It is therefore of interest to know if there is essential difference in the performances of the distance measures. Our experiments with histograms of single granularity (Table1) point to the direction that for category detection, the exponential $\chi^{2}$-kernel might be more suitable measure of histogram similarity than histogram intersection, although we did not explicitly evaluate this in the case of multiple granularities. It seems safe to say that at least the use of the $\chi^{2}$ distance does not make the multi-granularity kernel any weaker. This belief is supported by the anecdotal evidence of the $\chi^{2}$-distance and exponential kernels often working well in image processing applications. 
Table 1. Comparison of the MAP performance of $\chi^{2}$ and histogram intersection distance measures for single-granularity histograms

$$
\begin{array}{c|ccccccc} 
& \text { size } & & & & & & \\
& 128 & 256 & 512 & 1024 & 2048 & 4096 & 8192 \\
\hline \chi^{2} & 0.357 & 0.376 & 0.387 & 0.397 & 0.400 & 0.404 & 0.398 \\
\text { HI } & 0.333 & 0.353 & 0.359 & 0.367 & 0.387 & 0.380 & 0.381
\end{array}
$$

\subsection{Multi-granularity Kernel}

In Table 2 we show the classification performance of the multi-granularity kernel technique. The different columns correspond to combinations of increasing sets of histograms. In the experiments we use LBG codebooks with sizes from 128 to 8192 . The upper rows of the table correspond to different values of the weighting parameter $K$. The MAP values can be compared against the individual-granularity baseline values (row "indiv.") for the largest of the involved histograms, and also against the performance of post-classifier fusion of the histograms in question (row "fusion"). From the table one can observe that better performance is obtained by combining distances of multiple granularities already in the kernel calculation - just as the proposed technique does - rather than fusing the classifier outcomes later. Both methods for combining several granularities perform clearly better than the best one of the individual granularities. No weighting parameter value $K$ was found that would significantly outperform the unweighted sum of distances $(K=\infty)$.

In the tabulated experiments the speedup structure of Section 3 was not used. We repeated some of the experiments using the speedup structure with essentially no difference in MAP performance. The additional experiments also reveal that the inclusion of histograms larger than 8192 bins no longer improves the MAP.

\subsection{Histogram Smoothing}

For post-smoothing of histograms, we evaluated the category detection MAP for several values of $\lambda$ and $n_{\mathrm{nbr}}$. In the experiments the 2048 unit LBG histogram was used as a starting point. The best parameter value combination we

Table 2. MAP performance of the multi-granularity kernel technique

\begin{tabular}{l|cccccc}
$K$ & $128-256$ & $128-512$ & $128-1024$ & $128-2048$ & $128-4096$ & $128-8192$ \\
\hline 1 & 0.376 & 0.385 & 0.391 & 0.395 & 0.398 & 0.399 \\
2 & $\mathbf{0 . 3 8 2}$ & 0.394 & 0.402 & 0.409 & 0.413 & 0.414 \\
4 & $\mathbf{0 . 3 8 2}$ & $\mathbf{0 . 3 9 9}$ & 0.407 & 0.413 & 0.418 & 0.421 \\
$\infty$ & 0.379 & 0.396 & 0.409 & $\mathbf{0 . 4 1 8}$ & $\mathbf{0 . 4 2 3}$ & $\mathbf{0 . 4 2 5}$ \\
-4 & 0.377 & $\mathbf{0 . 3 9 9}$ & $\mathbf{0 . 4 1 1}$ & 0.417 & 0.422 & 0.422 \\
\hline indiv. & 0.376 & 0.387 & 0.397 & 0.400 & 0.404 & 0.398 \\
\hline fusion (BBR) & 0.380 & 0.396 & 0.404 & 0.409 & 0.414 & 0.415 \\
\hline
\end{tabular}


Table 3. MAP performance of different smoothing functions of the soft histogram technique for LBG codebook with 2048 codebook vectors

\begin{tabular}{l|ccccc} 
& $n_{\mathrm{nbr}}$ & & & & \\
& 3 & 5 & 8 & 10 & 15 \\
\hline inverse Euclidean & 0.426 & 0.427 & - & 0.421 & - \\
inverse squared Euclidean & 0.426 & 0.429 & 0.427 & - & \\
negexp $(\alpha \exp =3)$ & 0.428 & 0.433 & 0.435 & 0.435 & 0.433 \\
Gaussian $(\alpha \mathrm{g}=0.3)$ & 0.428 & 0.432 & 0.435 & 0.435 & 0.432
\end{tabular}

tried resulted in MAP 0.407 that is a slight improvement over the baseline MAP 0.400. The soft histogram technique, discussed next, provided clearly better performance which made more thorough testing of post-smoothing unappealing.

For the soft histogram technique, Table 3 compares the four different functional forms of smoothing functions for LBG codebook of size 2048. Among these, the exponential and Gaussian seem to provide somewhat better performance than the others. We evaluated the effect of the parameters $\alpha_{\exp }$ and $\alpha_{\mathrm{g}}$ to the detection performance and found the peak in performance to be broad in the parameter values. In these experiments, as well as in all subsequent ones, we use the value $n_{\mathrm{nbr}}=10$. The Gaussian functional form was chosen for the subsequent experiments of the two almost equally well performing functional forms of the exponential family.

In Table 4, a selection of MAP accuracies of the Gaussian soft histogram technique is shown for several different histogram sizes. The results for larger codebook sizes (512 and beyond) are obtained using the speed-up technique of Section 3. The results can be compared with the MAP of hard assignment baseline histograms on column "hard". It can be seen that the improvement brought by the soft histogram technique is substantial, except for the smallest histograms. This is intuitive since in small histograms the centers of the different histogram bins are far apart in the descriptor space and should therefore not be considered similar. For hard assignment histograms, the performance peaks with

Table 4. MAP performance of the soft histogram technique for different codebook sizes (rows) and different values of parameter $\alpha \mathrm{g}$ (columns)

\begin{tabular}{r|c|cccccc} 
& & $\alpha \mathrm{g}$ & & & & & \\
& hard & 0.05 & 0.1 & 0.2 & 0.3 & 0.5 & 1 \\
\hline 256 & 0.376 & - & - & 0.376 & 0.381 & 0.385 & 0.384 \\
512 & 0.388 & - & - & - & - & 0.406 & - \\
1024 & 0.393 & - & - & - & 0.419 & - & - \\
2048 & 0.400 & 0.423 & 0.429 & 0.433 & 0.435 & 0.433 & 0.423 \\
4096 & 0.403 & - & - & 0.438 & - & - & - \\
8192 & 0.395 & 0.443 & 0.445 & 0.448 & 0.445 & 0.434 & 0.419 \\
16384 & 0.392 & 0.450 & 0.451 & 0.451 & - & - & - \\
32768 & 0.387 & - & - & 0.448 & - & - & -
\end{tabular}


Table 5. The percentage of non-zero bin counts in various-sized histograms collected using either hard (conventional) or soft assignment to histogram bins

\begin{tabular}{l|rrrrrr} 
& 512 & 1024 & 2048 & 4096 & 8192 & 16384 \\
\hline Hard histograms & 53.47 & 35.33 & 21.45 & 12.11 & 6.72 & 3.63 \\
Soft histograms & 86.74 & 72.55 & 55.96 & 37.58 & 26.15 & 17.07
\end{tabular}

Table 6. MAP performance of combining soft histograms with the multi-granularity kernel technique

\begin{tabular}{l|ccccccc}
$\mathrm{K}$ & $128-256$ & $128-512$ & $128-1024$ & $128-4096$ & $128-8192$ & $128-16384$ & $128-32768$ \\
\hline 4 & 0.383 & 0.398 & 0.407 & 0.419 & 0.422 & 0.426 & 0.428 \\
$\infty$ & 0.377 & 0.395 & 0.408 & 0.427 & 0.432 & 0.437 & 0.442 \\
\hline indiv. & 0.385 & 0.406 & 0.419 & 0.438 & 0.448 & 0.451 & 0.448 \\
\hline fusion (BBR) & 0.385 & 0.405 & 0.416 & 0.433 & 0.442 & 0.447 & 0.447 \\
\hline
\end{tabular}

histograms of size 4096. The soft histogram technique makes larger histograms than this beneficial, the observed peak being at size 16384 .

The improved accuracy brought by the histogram smoothing techniques comes with the price of sacrificing some sparsity of the histograms. Table 5 quantifies this loss of sparsity. This could be of importance from the point of view of computational costs if the classification framework represents the histograms in a way that benefits from sparsity (which is not the case in our implementation).

Table 6 presents the results of combining soft histograms with the multigranularity kernel technique. From the results, it is evident that combining these two techniques does not bring further performance gain over the soft histograms. On the contrary, the MAP values of the combination are clearly lower than those of the largest soft histograms included in the combination (row "indiv.").

\section{Conclusions}

In this paper we have investigated methods of combining information in local feature histograms of several granularities in the descriptor space. The presented methods are such that the resulting histogram-like descriptors can be used as feature vectors in conventional vector space learning methods (here SVM), just as the histograms would be.

The methods have been evaluated in a set of image category detection tasks. By using the best one of the methods, a significant improvement of MAP from 0.404 to 0.451 was obtained in comparison with the best-performing histogram of a single granularity. Of the techniques, the soft assignment of descriptors to histogram bins resulted in clearly the best performance. Histogram smoothing as post-processing improved the performance only slightly over the singlegranularity histograms. The multi-granularity kernel technique was better than the baseline of single-granularity histograms with maximum MAP 0.425 , but 
clearly inferior to soft histograms. Combining soft histograms with the multigranularity kernel technique did not result in performance gain, supporting the conclusion that the both techniques leverage on the same information and are thus redundant. The soft histogram technique adds some computational cost in comparison with individual hard histograms as it becomes beneficial to use larger histograms, and the generated histograms are less sparse.

The issue of the generalisability of the described techniques is not addressed by the experiments of this paper. It seems plausible that this kind of smoothing methods would be usable also in other kinds of image analysis tasks and also with other local descriptors than just SIFT.

The selection of the parameters of the methods is another open issue. Currently we have demonstrated that there exists parameter values (such as $\alpha_{\mathrm{g}}$ in the soft histogram technique) that result in good performance. Finding such values has not been addressed here. Reasonably good parameter values could in practice be picked e.g. by cross-validation.

Of the discussed methods, the best performance was obtained by the soft histogram technique. However, the LBG codebooks for the histograms were generated with a conventional hard clustering algorithm. Using also here an algorithm specifically targeted at soft clustering instead - such as fuzzy c-means - could be beneficial. Yet, this is not so self-evident as the category detection performance is not the immediate target function optimised by the clustering algorithms.

\section{References}

1. Madigan, D., Genkin, A., Lewis, D.D.: BBR: Bayesian logistic regression software (2005), http://www. stat.rutgers. edu/ madigan/BBR/

2. Chang, C., Lin, C.: LIBSVM: a library for support vector machines (2001), http:// www.csie.ntu.edu.tw/ cjlin/libsvm

3. Everingham, M., Van Gool, L., Williams, C.K.I., Winn, J., Zisserman, A.: The PASCAL Visual Object Classes Challenge 2007 (VOC 2007) (2007), http://www . pascal-network.org/challenges/VOC/voc2007/workshop/index.html

4. Grauman, K., Darrell, T.: The pyramid match kernel: Efficient learning with sets of features. Journal of Machine Learning Research (2007)

5. Lowe, D.G.: Distinctive image features from scale-invariant keypoints. International Journal of Computer Vision 60(2), 91-110 (2004)

6. Mikolajcyk, K., Schmid, C.: Scale and affine point invariant interest point detectors. International Journal of Computer Vision 60(1), 68-86 (2004)

7. Viitaniemi, V., Laaksonen, J.: Improving the accuracy of global feature fusion based image categorisation. In: Falcidieno, B., Spagnuolo, M., Avrithis, Y., Kompatsiaris, I., Buitelaar, P. (eds.) SAMT 2007. LNCS, vol. 4816, pp. 1-14. Springer, Heidelberg (2007)

8. Viitaniemi, V., Laaksonen, J.: Experiments on selection of codebooks for local image feature histograms. In: Sebillo, M., Vitiello, G., Schaefer, G. (eds.) VISUAL 2008. LNCS, vol. 5188, pp. 126-137. Springer, Heidelberg (2008) 